\title{
First-principles prediction of kink-pair activation enthalpy on screw dislocations in bec transition metals: V, Nb, Ta, Mo, W, and Fe
}

\author{
L. Dezerald,${ }^{1, *}$ L. Proville, ${ }^{1}$ Lisa Ventelon, ${ }^{1}$ F. Willaime, ${ }^{1}$ and D. Rodney ${ }^{2}$ \\ ${ }^{1}$ CEA, DEN, Service de Recherches de Métallurgie Physique, F-91191 Gif-sur-Yvette, France \\ ${ }^{2}$ Institut Lumière Matière, Université Lyon 1, CNRS, UMR 5306, F-69622 Villeurbanne, France \\ (Received 19 January 2015; revised manuscript received 16 February 2015; published 9 March 2015)
}

\begin{abstract}
The atomistic study of kink pairs on screw dislocations in body-centered cubic (bcc) metals is challenging because interatomic potentials in bcc metals still lack accuracy and kink pairs require too many atoms to be modeled by first principles. Here, we circumvent this difficulty using a one-dimensional line tension model whose parameters, namely the line tension and Peierls barrier, are reachable to density functional theory calculations. The model parameterized in $\mathrm{V}, \mathrm{Nb}, \mathrm{Ta}, \mathrm{Mo}, \mathrm{W}$, and Fe, is used to study the kink-pair activation enthalpy and spatial extension. Interestingly, we find that the atomistic line tension is more than twice the usual elastic estimates. The calculations also show interesting group tendencies with the line tension and kink-pair width larger in group $\mathrm{V}$ than in group VI elements. Finally, the present kink-pair activation energies are shown to compare qualitatively with experimental data and potential origins of quantitative discrepancies are discussed.
\end{abstract}

DOI: 10.1103/PhysRevB.91.094105

PACS number(s): $62.20 . \mathrm{F}-$

\section{INTRODUCTION}

The thermally activated glide of $1 / 2\langle 111\rangle$ screw dislocations controls the temperature-dependent plasticity of body-centered-cubic (bcc) metals [1,2]. Intense investigations are lead on these dislocations (see for example Refs. [3-17]), the main issue being to derive the dislocation mobility law from the atomic scale [18]. Among the different computational studies, calculations based on density-functional theory (DFT) have established some important features that were previously matter of debate in bcc transition metals, such as the nondegenerate dislocation core structure $[6,10,17]$, the $\{110\}$ glide plane [9], and the single-hump Peierls barrier [10,17,19-21]. However, the main drawback of DFT-based methods applied to extended defects such as dislocations is the limited number of atoms that can be considered in the simulation cell, typically a few hundred. These cell sizes are sufficient to study straight dislocations perfectly aligned with their $\mathbf{b}=a_{0} / 2\langle 111\rangle$ Burgers vector, where $a_{0}$ is the equilibrium lattice parameter, because the translational invariance along the dislocation line allows to reduce the cell size in this direction to a slab of length $b=|\mathbf{b}|=a_{0} \sqrt{3} / 2$ using periodic boundary conditions [9-12]. At finite temperatures [22], however, the motion of screw dislocations proceeds through the formation of kink pairs, thereby breaking the translational invariance along the dislocation line. Simulating this process at the atomic scale thus requires to consider a long dislocation with kinks, which is out of the reach of current DFT calculations. It is possible to circumvent this difficulty by employing the line tension (LT) model, where the dislocation is represented by a one-dimensional (1D) elastic line on a substrate, which is a standard approach in dislocation theory, referred to as the 1D Frenkel-Kontorova model [23-25], the string model [26], or more recently, the line-on-substrate model [27]. It is also often simply named the line tension (LT) model [28,29].

\footnotetext{
*Present address: Department of Civil and Environmental Engineering, Massachusetts Institute of Technology, MultiScale Material Science for Energy and Environment, UMI 3466 CNRS-MIT, 77 Massachusetts Avenue, Cambridge, Massachusetts 02139, USA.
}

The Hamiltonian of the LT model is composed of two distinct parts: (i) the substrate potential, also called Peierls potential and (ii) the elastic energy associated with the stiffness of the dislocation line, i.e. the line tension. To derive the first term, DFT computations in a $1 b$ slab cell can be used, while the computation of the second term requires us to break the translational invariance along the dislocation line. A methodology has recently been developed to determine the line tension with simulation cell sizes accessible to DFT calculations using specific constrained displacements of atoms situated inside the dislocation core [30]. The validity of this method has been verified using three different semiempirical interatomic potentials and it has then been applied to iron using DFT [30].

In the present study, we extend this initial approach to compute kink pairs in all bcc transition metals: group $\mathrm{V}$ vanadium, niobium, and tantalum, group VI molybdenum and tungsten, and ferromagnetic iron. The values obtained for the line tension are compared to continuous elasticity theory. The LT model is then used to predict the kink-pair activation enthalpy and the kink-pair profile on screw dislocations, which are otherwise beyond the reach of direct DFT calculations. Eventually, we compare the DFT-based results to available experimental data, based on either cyclic deformation [22,31,32] or successive tensile deformation techniques [33-35].

\section{SIMULATION CONDITIONS}

The DFT simulation cell is oriented such that the $\{110\}$ screw dislocation glide plane has a normal vector along the $Z=[1 \overline{1} 0]$ direction with the dislocation line along the $X=$ [111] direction and the glide direction along the $Y=[\overline{1} \overline{1} 2]$ axis. We consider a quadrupolar geometry, where a dislocation dipole is introduced in a simulation cell with periodic boundary conditions in all three directions [10].

The first-principles electronic structure calculations were performed within the DFT framework using the PWSCF plane-wave code [36]. The pseudopotentials are ultrasoft with semicore electrons for $\mathrm{V}, \mathrm{Nb}, \mathrm{Ta}, \mathrm{Mo}$, and $\mathrm{W}$, and without semicore electron but with nonlinear core corrections for $\mathrm{Fe}$. 
These pseudopotentials have been tested and used in previous studies [21,37-39]. $1 \times 2 \times 16$ and $1 \times 2 \times 8$ shifted $k$-point grids were used for the $1 b$ and $2 b$ cells respectively. This $k$-point density is close to that of a $16 \times 16 \times 16$ grid for a one-atom bcc cell. As pointed out in Ref. [40], it is too sparse for accurate calculations of bulk properties like the $C_{44}$ elastic constants in $\mathrm{V}$ and $\mathrm{Nb}$ due to the presence of a van Hove singularity near the Fermi level [41]. However, since this singularity is smeared out by the dislocation [21], this $k$-point density issue should not affect the present results. Residual forces after relaxation were smaller than $10^{-3} \mathrm{eV} / \AA$, and the convergence criterion for self-consistency on the total energy was $10^{-7} \mathrm{eV}$. The Hermite-Gauss scheme to broaden the electronic density of states was used with a smearing of $0.3 \mathrm{eV}$. The calculations in iron are spin polarized [42] (ferromagnetic iron) and the Perdew-Burke-Ernzerhof generalized gradient approximation (GGA) was used with a wave-function cutoff of $40 \mathrm{Ry}$. The bulk properties of all investigated elements calculated with these pseudopotentials are presented in Ref. [21].

\section{LINE TENSION MODEL}

\section{A. Formulation and approximations}

In the LT model, the screw dislocation is represented by a single function $y(x)$, defined as the position $y$ in the glide plane of the dislocation segment situated at the $x$ coordinate along the dislocation line. If we assume that the derivatives of the function $y(x)$ are small and furthermore that the Peierls barrier is small compared to the dislocation self-energy, we can write a linearized version of the LT model $[27,43,44]$, where the dislocation enthalpy $H_{L T}$ is expressed as:

$$
H_{\mathrm{LT}}\left(y, \sigma_{\mathrm{xz}}\right)=\int d x\left[V_{P}(y(x))-\sigma_{\mathrm{xz}} b y(x)+\frac{\Gamma}{2}\left(\frac{d y}{d x}\right)^{2}\right] \text {. }
$$

Here, $\Gamma$ is the line tension, which is assumed to be independent of the dislocation line orientation in this simple model, $V_{P}$ the Peierls potential and $\sigma_{\mathrm{xz}}$ the applied stress. In this study, we neglect the dependence of the dislocation Peierls potential on the applied stress. It should be noted that the line tension model does not account for the long-range elastic interaction between kinks, which have an edge character, nor does it account for the asymmetry between left and right kinks [45]. We have however checked by comparison with atomistic simulations using EAM potentials [30] that these effects have no consequence for the nucleation of kink pairs at high stress as considered here.

In order to combine the LT model with atomistic simulations, the integral in Eq. (1) must be discretized. The dislocation line is thus decomposed into elementary segments of length $b$, the position of which, $\left\{Y_{n}\right\}$, is computed using the methodology explained in Ref. [30]. After discretization, Eq. (1) becomes:

$$
\begin{aligned}
H_{\mathrm{LT}}\left(\left\{Y_{n}\right\}, \sigma_{\mathrm{xz}}\right)= & b \sum_{n}\left[V_{P}\left(Y_{n}\right)-\sigma_{\mathrm{xz}} b Y_{n}\right. \\
& \left.+\frac{\Gamma}{2 b^{2}}\left(Y_{n+1}-Y_{n}\right)^{2}\right]
\end{aligned}
$$

where the sum over subscript $n$ implicitly accounts for the periodic boundary condition in the $X$ direction. The Hamiltonian in Eq. (2) corresponds to the 1D Frenkel-Kontorova model if the substrate potential is sinusoidal [25]. The main interest of such a discretized model is that the different terms involved in Eq. (2) can all be computed from atomistic simulations as described in the following. Since the variable $Y_{n}$ corresponds to the $n$th dislocation segment position, we define the reaction coordinate as $Y_{n} / L_{P}$, with $L_{P}=a_{0} \sqrt{2 / 3}$ the distance between nearest valleys of the Peierls potential. When the dislocation goes from one Peierls valley to the next, this reduced coordinate varies between 0 and 1, with the initial position of the straight dislocation taken as a reference.

\section{B. Peierls barriers}

The Peierls barrier, $V_{P}$, was computed at the atomistic level from the hypothetical situation of a straight infinite dislocation moving between two Peierls valleys. All atoms in a given [111] atomic column are thus forced to move rigidly, with the same displacement. The Peierls barrier is then identified with the dislocation minimum energy path (MEP) between Peierls valleys.

The DFT calculations were performed in a cell of $1 b$ in the $X$ direction, containing 135 atoms within the quadrupolar arrangement. Lengths in the $Y$ and $Z$ directions are $15 L_{P}$ and $9 a_{0} / \sqrt{2}$, respectively. The reaction coordinate method (or drag method) [46] is used to determine the Peierls barrier with eleven images. This method was shown in Ref. [19] to be equivalent to the nudged elastic band method for the simple path considered here and is preferred for DFT calculations because of its smaller computational cost. The results are reported in Figs. 1(a)-1(c) for the different elements, as a function of $Y_{1} / L_{P}$, the normalized dislocation position along the path (the subscript " 1 " indicates that only one slab is used in the $X$ direction) [30]. The calculated energies are identical to those from Ref. [21], but the definition for the dislocation position is different. Here we use a one-dimension disregistry that gives the dislocation position in the $Y$ direction. The drawback of this definition is that the final position for the dislocation is not exactly equal to 1 in our computations. This arises from the fact that the dislocations constituting the dipole interact with each other, given the small distance $\left(7.5 L_{P}\right)$ between them. This artifact leads to Peierls stresses values slightly higher than the ones predicted in Ref. [21] where two different methods were used to evaluate the dislocation position. However, the error remained less than $25 \%$, which is similar to the uncertainty related to the definition of the dislocation position pointed out in Ref. [21]. In Figs. 1(a)-1(c), continuous lines stem from a cubic spline procedure and correspond to the function $V_{P}$ used in Eq. (2).

\section{Line tension}

To compute the line tension, $\Gamma$, a dislocation bow out has to be considered in the simulation cell and thus, the translational symmetry along the dislocation line must be broken. To this end we considered a cell $2 b$ thick in the $X$ direction. The dimensions in the $Y$ and $Z$ directions are unchanged and the cell is duplicated in the $X$ direction, with a total number of 

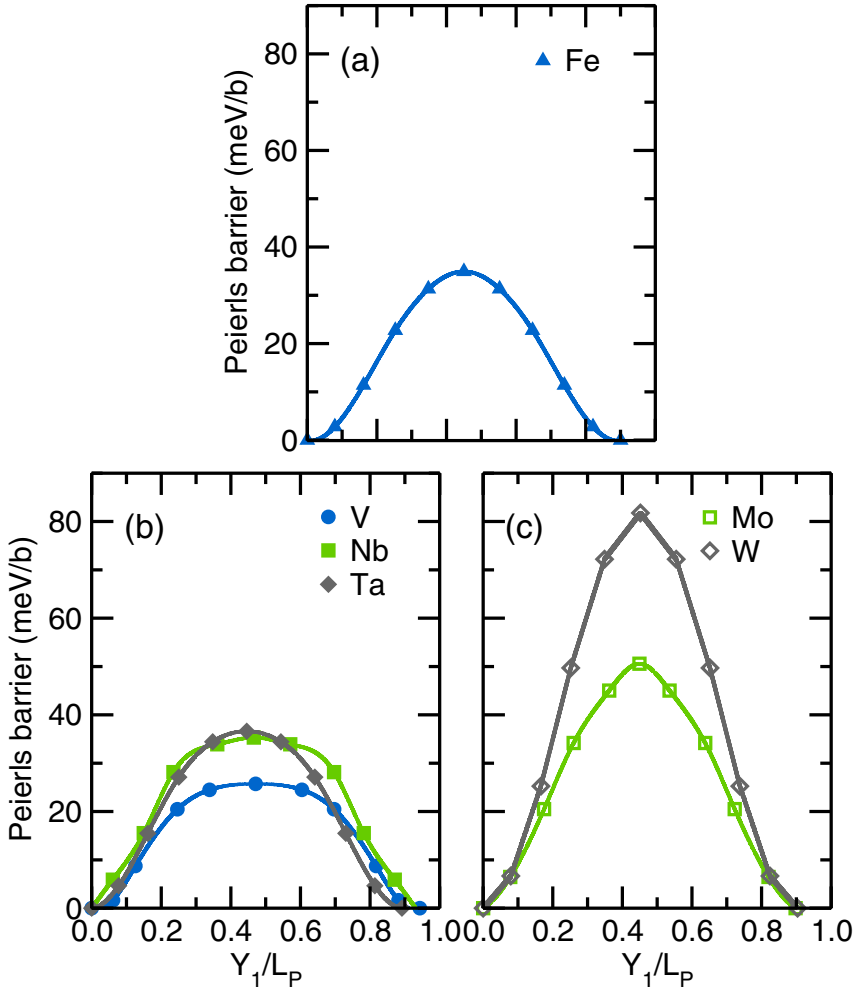

FIG. 1. (Color online) Minimum energy path between two Peierls valleys per unit of $b$ for a straight screw dislocation computed with DFT for different bcc metals: (a) Fe, (b) group V elements: V, $\mathrm{Nb}$, Ta, and (c) group VI elements: Mo, W. The lines are the cubic splines used to define the function $V_{P}(Y)$ in Eq. (2).

atoms $N_{\text {at }}=270$. When a straight dislocation changes Peierls valley, the three [111] atomic columns around the intermediate hard core undergo much larger displacements than the rest of the crystal. Applying constrained displacements to these atoms, as described in Ref. [30], we can enforce a bow out on the dislocation consistent with the first stage of formation of a kink pair and compute the associated energy.

In this process, a dislocation segment starts to glide towards the next Peierls valley, while the rest of the dislocation line remains in the initial Peierls valley. To mimic this process, we considered a dislocation relaxed under zero stress and constrained the core atoms situated in one of the two slabs constituting the $2 b$ cell to follow a prescribed trajectory, while the atoms in the second slab were fixed to their initial positions along the $X$ direction. In this way, we impose different dislocation positions $Y_{1}$ and $Y_{2}$ in the two slabs. The energy difference, $\Delta E$, between this constrained state and the relaxed initial state, as well as the position difference $Y_{2}-Y_{1}$ were then calculated. According to Eq. (2), and accounting for periodic boundary conditions along the $X$ direction, we have:

$$
\Delta E_{\mathrm{LT}}=\Delta E-b V_{P}\left(Y_{1}\right)-b V_{P}\left(Y_{2}\right)=\Gamma / b\left(Y_{2}-Y_{1}\right)^{2} .
$$

This function is quadratic in $\left(Y_{2}-Y_{1}\right)$ with a curvature equal to $2 \Gamma / b$, allowing us to fit the line tension, denoted $\Gamma_{\mathrm{DFT}}$ thereafter.

We report the variation of $\Delta E_{\mathrm{LT}}$ against $\left(Y_{2}-Y_{1}\right) / L_{P}$ as symbols in Figs. 2(a)-2(c), along with the quadratic fits, as

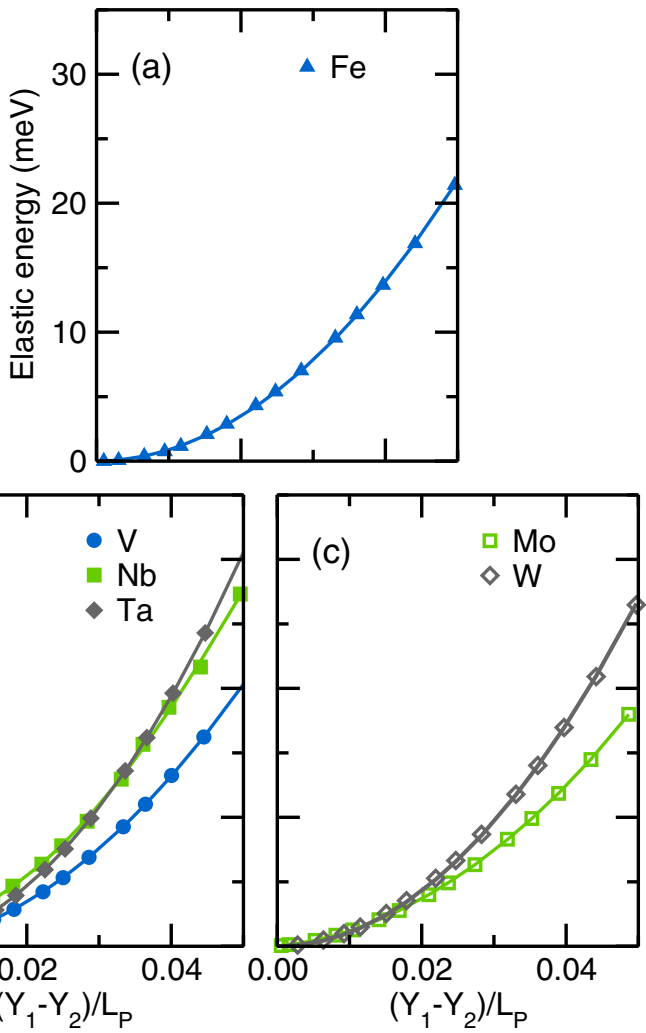

FIG. 2. (Color online) Elastic energy of the dislocation computed from DFT in the $2 b$ slab cell (symbols) and from a LT model (lines) for (a) Fe, (b) group V elements, and (c) group VI elements. On the $X$ axis, $Y_{1}$ and $Y_{2}$ correspond to the position of the dislocation segments in the first and second $1 b$ slab, respectively. $L_{P}$ is the distance between two Peierls valleys. The adjusted values of the line tension $\Gamma_{\mathrm{DFT}}$ are reported in Table I.

continuous lines. The fits are very satisfactory in the interval $\left(Y_{2}-Y_{1}\right) / L_{P} \in[0,0.05]$, for all investigated elements and lead to the values of $\Gamma_{\text {DFT }}$ reported in Table I.

We then compared these LT predictions to the LT values predicted from isotropic and anisotropic elasticity. The isotropic elastic value of the line tension is given by:

$$
\Gamma_{\mathrm{ela}}^{\mathrm{iso}}=\frac{\mu b^{2}}{4 \pi} \frac{1+v}{1-v} \ln \left(\frac{R}{r_{0}}\right),
$$

TABLE I. Line tension (in eV/Å) computed from DFT $\left(\Gamma_{\mathrm{DFT}}\right)$ and from isotropic and anisotropic elasticity theory $\left(\Gamma_{\text {ela }}^{\mathrm{iso}}\right.$ and $\Gamma_{\text {ela }}^{\text {aniso }}$, respectively). The total line tension, $\Gamma_{\text {tot }}^{\text {aniso }}$, takes into account both the core energy and core field contributions.

\begin{tabular}{lcccc}
\hline \hline & $\Gamma_{\text {DFT }}$ & $\Gamma_{\text {ela }}^{\text {iso }}$ & $\Gamma_{\text {ela }}^{\text {aniso }}$ & $\Gamma_{\text {tot }}^{\text {aniso }}$ \\
\hline $\mathrm{V}$ & 3.17 & 0.68 & 0.37 & 0.51 \\
$\mathrm{Nb}$ & 4.02 & 0.44 & 0.18 & 0.37 \\
$\mathrm{Ta}$ & 4.22 & 1.72 & 1.97 & 2.13 \\
$\mathrm{Mo}$ & 2.86 & 1.93 & 1.58 & 2.03 \\
$\mathrm{~W}$ & 3.89 & 2.56 & 2.55 & 3.10 \\
$\mathrm{Fe}$ & 3.52 & 1.51 & 2.05 & 2.30 \\
\hline \hline
\end{tabular}


where $\nu$, the Poisson ratio and $\mu$, the shear modulus on $\{110\}$ are calculated by imposing that the prelogarithmic factor of the elastic energy for a straight dislocation obtained from the anisotropic and isotropic elasticity theory are equal for both the screw and the edge orientations, as suggested by Scattergood and Bacon [47,48]. The outer and inner cut-off radii $R$ and $r_{0}$ are chosen equal to the kink-pair width, $W$ and the Burgers vector $b$, respectively. The choice of $R=W$ relies on the line tension computation presented in Ref. [49]. Here, the kink-pair width was calculated at finite but low stress, $\sigma=$ $40 \mathrm{MPa}$, which corresponds to a typical value of the athermal stress. The anisotropic LT, $\Gamma_{\text {ela }}^{\text {aniso }}(\theta)$, was calculated within Stroh formalism [50] using the DISDI code [51].

In order to compare the line tension extracted from DFT to the LT values from elasticity theory, we calculated a third value for the LT, $\Gamma_{\text {tot }}^{\text {aniso }}$, that takes into account the core energy $E_{\text {core }}$, as well as the dilatation field contribution $E_{\text {dil }}$ observed in DFT calculations [21,52]. $\Gamma_{\text {tot }}^{\text {aniso }}$ is expressed as follows:

$$
\Gamma_{\text {tot }}^{\text {aniso }}=\Gamma_{\text {ela }}^{\text {aniso }}(\theta)+E_{\text {core }}+E_{\text {dil }},
$$

where $\theta$ is the angle between the dislocation line direction and Burgers vector. In Eq. (5), we neglect the dependence of $E_{\text {core }}$ and $E_{\mathrm{dil}}$ on $\theta$, and we do not take into account the core traction term in the Volterra elastic field contribution, which was shown to be negligible compared to the Volterra cut contribution [12]. The values of $E_{\text {core }}$ and $E_{\text {dil }}$ were calculated with DFT in a previous study [21].

According to the present model, the LT values predicted using elasticity are much smaller than those from DFT. This illustrates the limitation of applying elasticity to highly localized processes like kink-pair formation, which involves dislocation segments with a length comparable to the core radius [30,53]. Interestingly, the difference between the isotropic and anisotropic elastic LT values is much smaller than the difference between the elastic and the DFT LT values. This confirms that the discrepancy does not stem from anisotropy but that it is related to the atomistic nature of the process. Note that the DFT values are calculated at zero stress, while the kinkpair widths used for the elastic LT evaluation were calculated at $\sigma=40 \mathrm{MPa}$, but we checked that this difference cannot compensate for the large discrepancies discussed earlier.

In order to study the effect of electronic band filling, the values of $\Gamma_{\mathrm{DFT}}$ and $\Gamma_{\text {ela }}$ were normalized by $E_{\mathrm{coh}} / b$ (Fig. 3), where $E_{\text {coh }}$ is the cohesive energy calculated in Ref. [21]. The calculation of the cohesive energy reported in Ref. [21] requires the energy of the isolated atom, which was obtained from spin-polarized calculations using a cubic box of side length $21 \AA$ and one $k$ point. As expected, the normalized $\Gamma_{\text {ela }}$ reproduce the classical group dependence on band filling with normalized values larger in group VI than in group V elements [54]. Surprisingly, this dependence is reversed for the DFT line tensions, where the normalized $\Gamma_{\mathrm{DFT}}$ are slightly larger in group V than in group VI elements. We also notice that the line tension in $\mathrm{Fe}$ is closer to group $\mathrm{V}$ than group VI elements. In a previous paper [21], we showed that the normalized Peierls energy, $E_{\mathrm{P}} /\left(E_{\mathrm{coh}} / b\right)$ is twice larger in group VI than in group V elements, and intermediate in Fe. This trend is reversed for the DFT LT values, and moreover, we should note that the difference between DFT normalized

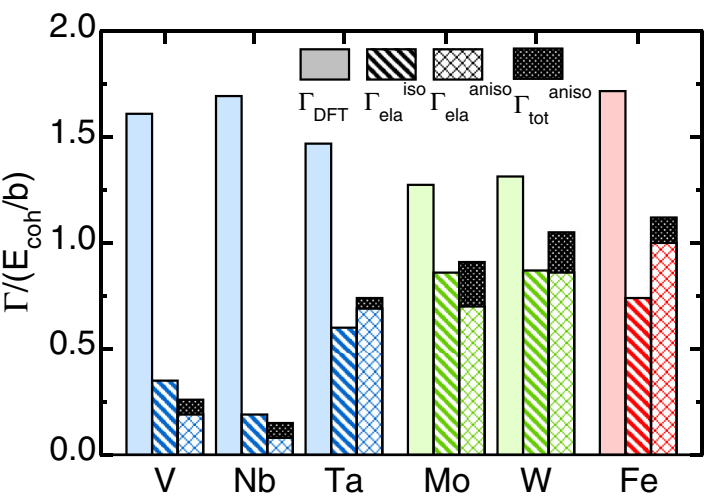

FIG. 3. (Color online) Normalized line tension computed from DFT and from isotropic (iso) and anisotropic (aniso) continuous elasticity. $\Gamma_{\text {tot }}^{\text {aniso }}$ takes into account the dislocation core energy and dilatation field contributions.

LT values is much smaller than other normalized quantities such as the Peierls energy.

\section{KINK-PAIR ACTIVATION ENTHALPY AND KINK-PAIR PROFILE}

An excellent agreement on the kink-pair formation enthalpy between LT predictions and direct atomistic calculations using three different interatomic models was reported in Ref. [30]. This validated the initial approach and showed that the potential variation of the line tension between the bottom and the top of the Peierls landscape can be neglected. Despite the differences that have been found for the dislocation trajectory in the different bcc metals [21], our computation of the line tension, based on the linear interpolation of the atomic trajectories [30], remains valid since it has been tested on different interatomic potentials $[16,62,63]$ that predict very different dislocation paths in Fe. We may therefore use the DFT computations of the Peierls barriers and line tensions to predict the kink-pair formation enthalpy in all investigated bcc metals. In order to compute the kink-pair solution, we chose an analytical form of the kink pair as a starting configuration and we applied a Newton-Raphson procedure to the functional presented in Eq. (2) in order to find the closest extremal configuration. The trial solution has the form

$$
\begin{aligned}
Y_{i}= & L_{P}\left[1+\tanh \left(\alpha\left(i-n_{-}\right)\right)\right] / 2 \\
& -L_{P}\left[1+\tanh \left(\alpha\left(i-n_{+}\right)\right)\right] / 2,
\end{aligned}
$$

where $\alpha, n_{-}$, and $n_{+}$are parameters adjusted to build a starting configuration close enough to the saddle state. The results for the kink-pair enthalpy versus applied stress are reported in Figs. 4(a)-4(c). In Table II, we reported the Peierls stress, i.e., the stress at which the kink-pair enthalpy vanishes, and the kink-pair formation energy, which corresponds to the kink-pair formation enthalpy at zero stress, for all investigated metals. Table II evidences the well-known discrepancy between calculated and experimental Peierls stresses. This discrepancy was shown to arise from zero-point energy vibrations [16] and nonglide effects [64] that were not taken into account in the present study because they remain a computational challenge within DFT. 

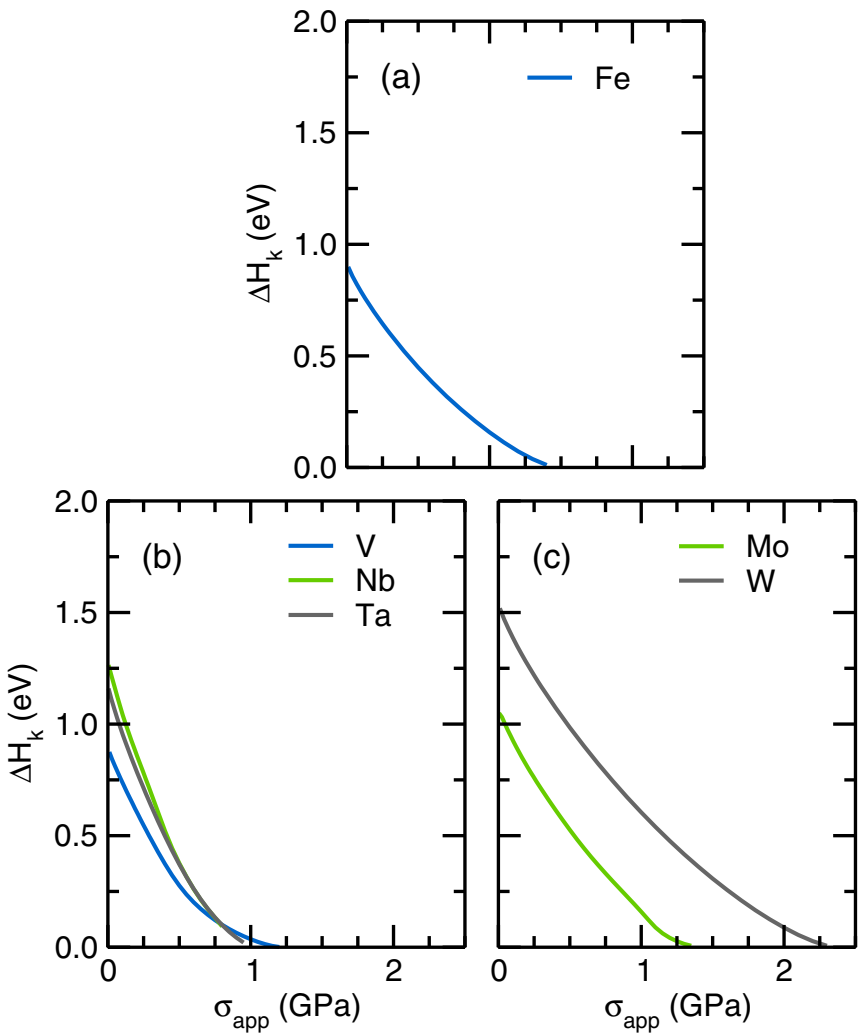

FIG. 4. (Color online) Kink-pair activation enthalpy computed from the LT model, parametrized with DFT for (a) Fe, (b) group $\mathrm{V}$ elements, and (c) group VI elements.

From the LT calculations, we also obtained the profile of the critical kink pair on the screw dislocation at $\sigma=40 \mathrm{MPa}$ [see Figs. 5(a)-5(c)], which we used to determine the kink-pair width in Table II. The kink-pair widths reported in Table II are defined as $W=\left(d_{1}+d_{2}\right) / 2$, where $d_{1}$ and $d_{2}$ are the widths between the intersections of the tangents at the inflexion point and the tangents of the profiles in their Peierls valleys, respectively. The method used to determine $d_{1}$ and $d_{2}$ is illustrated in Fig. 5(a). In Fig. 5, the kink-pair height (respectively, the kink-pair width) was normalized by the distance between nearest Peierls valleys, $L_{P}$ (respectively by the Burgers vector,

TABLE II. DFT computed kink-pair features derived from Figs. 4(a)-4(c) and 5(a)-5(c): the Peierls stress, $\sigma_{P}^{\text {DFT }}$ (in MPa), the kink-pair energy, $\Delta E_{k}^{\mathrm{DFT}}$ (in eV) and the kink-pair width, $W$ (in b units), estimated at $\sigma=40 \mathrm{MPa}$ using the method presented in Fig. 5. We also reported the experimental data for the Peierls stress, $\sigma_{P}^{\exp }$ (in $\mathrm{MPa}$ ) and for the kink-pair energy, $\Delta E_{k}^{\text {exp }}$ (in eV), taken at the lowest measured stress value in regime I (see Sec. V for details).

\begin{tabular}{lrcccc}
\hline \hline & $\sigma_{P}^{\text {DFT }}$ & $\sigma_{P}^{\exp }$ & $\Delta E_{k}^{\text {DFT }}$ & $\Delta E_{k}^{\exp }$ & $W$ \\
\hline $\mathrm{V}$ & 1210 & $360[55]$ & 0.89 & & 19.5 \\
$\mathrm{Nb}$ & 890 & $450[56]$ & 1.28 & $0.62[22]$ & 21.5 \\
$\mathrm{Ta}$ & 1030 & $350[57]$ & 1.17 & $0.83[31]$ & 20.8 \\
$\mathrm{Mo}$ & 1390 & $870[58]$ & 1.05 & $1.19[32]$ & 15.9 \\
$\mathrm{~W}$ & 2340 & $900[59]$ & 1.54 & $2.05[33]$ & 15.6 \\
$\mathrm{Fe}$ & 1450 & $370[60,61]$ & 0.91 & $0.83[35]$ & 19.3 \\
\hline \hline
\end{tabular}

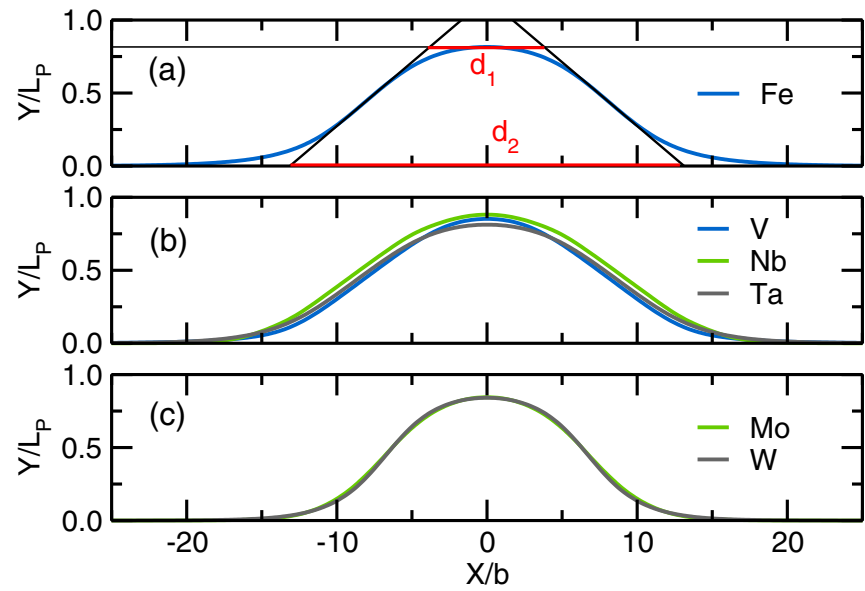

FIG. 5. (Color online) Profiles of the dislocation with a critical kink pair at $\sigma=40 \mathrm{MPa}$, computed from the LT model with parameters deduced from DFT calculations in (a) Fe, (b) group V elements, and (c) group VI elements.

b) to enable comparison between elements. A group trend is evidenced on the width of the kink-pair (see Table II): in group $\mathrm{V}$ elements, the kink-pair width is larger than in group VI elements, while the width is intermediate in $\mathrm{Fe}$.

Figure 6 represents the kink-pair energy, $\Delta E_{k}^{\mathrm{DFT}}$, as a function of the energy parameter, $\Delta E_{F K}=4 \sqrt{2} / \pi \times L_{P} \sqrt{\Gamma V_{P}}$, which scales the kink-pair energy in an analytical calculation assuming a cosinelike Peierls potential and small variations of $Y_{n}$ with respect to position $n$ along the $1 \mathrm{D}$ chain in Eq. (2), i.e., the continuous version of the Frenkel-Kontorova model, also known as the Sine-Gordon chain [26]. In this figure, we also reported the results from a previous study, where different interatomic models for Fe were employed and compared to other DFT-based computations [30]. Note that there is an

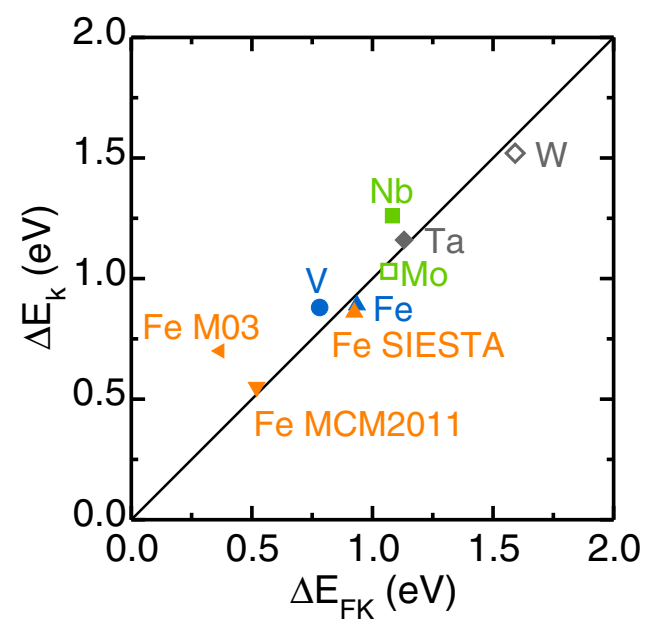

FIG. 6. (Color online) Kink-pair formation energy as a function of $\Delta E_{F K}=4 \sqrt{2} / \pi \times L_{P} \sqrt{\Gamma V_{P}}$ for all investigated elements. The orange symbols correspond to the values obtained previously in $\mathrm{Fe}[30]$ with either the SIESTA code, the interatomic potential MCM2011 [16] or the interatomic potential M03 [62]. The solid line has a slope of 1, as predicted from the LT model based on a cosine function for the Peierls potential. 
excellent agreement between the present DFT calculation with PWSCF and the previous DFT calculations obtained with the SIESTA code in Fe reported in Ref. [30].

One can notice in Fig. 6 that all the DFT calculated kink-pair energies are situated in the vicinity of a straight line with a slope equal to 1 , as predicted by the analytical calculation. Deviations from this line come from the nonsinusoidal shape of the energy profiles. The point that deviates most from the straight line in Fig. 6 corresponds to the Mendelev interatomic Fe model [62], as expected since this potential has a doublehumped Peierls barrier [7,10]. V and Nb, for which the Peierls barriers are rather flat [Fig. 1(b)], correspond to kink-pair energies situated further from the line than Ta, Mo, W, and Fe that have Peierls barriers closer to cosine [Figs. 1(a), 1(c)].

In the work of Marinica et al. [65], the kink-pair formation energy computed with three different EAM potentials for $\mathrm{W}$ was found to scale with $\sqrt{V_{P}}$, which according to the present scaling and the fact that all three potentials have the same distance between Peierls valleys, implies that they also have similar line tensions. Assuming that first-principles calculations would also yield the same line tension, the scaling was used to predict kink-pair formation energies from the only knowledge of first-principles Peierls barriers. The values obtained here are slightly different $\left(\Delta E_{k}=1.54 \mathrm{eV}\right.$ in W here while in Ref. [65], $\Delta E_{k}=2.1 \mathrm{eV}$ ), but the present work is more accurate since we compute separately the Peierls barrier and kink-pair formation energy and do not need any assumption on the line tension.

\section{DISCUSSION}

The DFT kink-pair formation energies are compared to experimental data in Table II. Although there is a general agreement, discrepancies remain. A quantitative comparison between DFT and experiments is however difficult for several reasons, related to both experimental and computational aspects. First, in the experiments that give access to the resolved shear stress, $\sigma$, as a function of the strain rate, $\dot{\varepsilon}$, and the temperature, $T$, three different regimes are usually identified, regime I (low $\sigma$, high $T$ ), regime II (intermediate $\sigma$ and $T$ ), and regime III (large $\sigma$, low $T$ ). In Refs. [33-35], these three regimes lead to three different predictions of the kink-pair formation enthalpy as a function of stress, and in turn, three different kink-pair formation energies, since the enthalpy curves do not cross the zero-stress axis at the same energy [33-35]. Moreover, the extrapolations involve analytical models using line tensions taken from the elasticity theory, which strongly underestimates the DFT values, as shown in Sec. III. To avoid such treatments, we reported here the kink-pair formation enthalpies measured at the lowest stresses available [22,31-33,35], without any additional theoretical assumption. These values necessarily underestimate the kink-pair formation energies and are slightly below those generally accepted and compiled for instance in Ref. [66]. The latter however probably overestimates the kink-pair formation energies because of the analytical extrapolation, which increases rapidly at low stresses. From the difference between these data, we can infer that the experimental uncertainty is between 0.05 and $0.1 \mathrm{eV}$.

Another difficulty is that the experimental data were obtained at high temperatures (regime I), while our DFT computations do not account for finite-temperature effects. The first missing contribution is the vibrational entropy, which was recently shown to significantly lower the Peierls free energy barrier at temperatures larger than room temperature in Fe [67]. This decrease is related to the anharmonicity of the atomic interactions and as such, this effect can be expected to concern all materials, although its generality has yet to be demonstrated. The electronic entropy could also play a role, since the local density of states around the Fermi level significantly changes upon defect formation or displacement in these metals $[21,68,69]$.

\section{SUMMARY AND CONCLUSIONS}

The dislocation line tension was calculated from DFT in six different bec transition metals. The present work is readily applicable to screw dislocations in all bcc metals and could even be extended to other dislocations in more complex materials provided setting up a method to mimic the formation of a kink pair [30]. Once parametrized with DFT-based computations for the line tension and Peierls barrier, the LT model allows to predict the kink-pair formation enthalpy at different applied stresses. We obtained the Peierls stress and the kink-pair formation energy, as reported in Table II and we showed that both isotropic and anisotropic elasticity strongly underestimate the DFT-based line tension. This important result raises questions on the interpretations of deformation tests [22,31-35], which often rely on models where the elasticity theory is used to determine the line tension.

The DFT-based calculations for the kink-pair formation enthalpy have been compared to measured data at the lowest applied stress. Discrepancies have been evidenced and require further investigation, possibly in the direction of vibrational [67] or electronic entropy which we intend to explore in future work.

\section{ACKNOWLEDGMENTS}

The authors would like to acknowledge support from the Partnership for Advanced Computing in Europe (PRACE) for awarding us access to resource MareNostrum III based in Spain at the Barcelona Supercomputing Center (BSC) within the DIMAIM project. The authors acknowledge J. Douin (CNRS, CEMES, France) for providing the DISDI code [51] used to calculate the LT values from elasticity theory. This work has been carried out within the framework of the EUROfusion Consortium and has received funding from the Euratom research and training programme 20142018 under Grant Agreement No. 633053. The views and opinions expressed herein do not necessarily reflect those of the European Commission.

[3] S. Ismail-Beigi and T. A. Arias, Phys. Rev. Lett. 84, 1499 (2000).

[4] A. H. W. Ngan and M. Wen, Phys. Rev. Lett. 87, 075505

(2001).
[2] D. Caillard and J. L. Martin, Thermally Activated Mechanisms of Crystal Plasticity (Pergamon, Oxford, 2003). 
[5] L. H. Yang, P. Söderlind, and J. A. Moriarty, Philos. Mag. A 81, 1355 (2001).

[6] S. L. Frederiksen and K. W. Jacobsen, Philos. Mag. 83, 365 (2003).

[7] J. Chaussidon, M. Fivel, and D. Rodney, Acta Mater. 54, 3407 (2006).

[8] L. Ventelon, F. Willaime, and P. Leyronnas, J. Nucl. Mater. 386-388, 26 (2009).

[9] C. Woodward and S. I. Rao, Phys. Rev. Lett. 88, 216402 (2002).

[10] L. Ventelon and F. Willaime, J. Comput,-Aided Mater. Des. 14, 85 (2007).

[11] L. Ventelon and F. Willaime, Philos. Mag. 90, 1063 (2010).

[12] E. Clouet, L. Ventelon, and F. Willaime, Phys. Rev. B 84, 224107 (2011).

[13] P. A. Gordon, T. Neeraj, Y. Li, and J. Li, Modell. Simul. Mater. Sci. Eng. 18, 085008 (2010).

[14] C. Domain and G. Monnet, Phys. Rev. Lett. 95, 215506 (2005).

[15] L. Romaner, C. Ambrosch-Draxl, and R. Pippan, Phys. Rev. Lett. 104, 195503 (2010).

[16] L. Proville, D. Rodney, and M.-C. Marinica, Nature Mater. 11, 845 (2012).

[17] C. R. Weinberger, G. J. Tucker, and S. M. Foiles, Phys. Rev. B 87, 054114 (2013).

[18] E. Orowan, Proc. Phys. Soc. 52, 8 (1940).

[19] L. Ventelon, E. Clouet, F. Willaime, and D. Rodney, Acta Mater. 61, 3973 (2013).

[20] M. Itakura, H. Kaburaki, and M. Yamaguchi, Acta Mater. 60, 3698 (2012).

[21] L. Dezerald, L. Ventelon, E. Clouet, C. Denoual, D. Rodney, and F. Willaime, Phys. Rev. B 89, 024104 (2014).

[22] A. Seeger and U. Holzwarth, Philos. Mag. 86, 3861 (2006).

[23] J. Frenkel and T. Kontorova, J. Phys.-USSR 1, 137 (1939).

[24] B. Joós, Solid State Commun. 42, 709 (1982).

[25] B. Joós and M. S. Duesbery, Phys. Rev. B 55, 11161 (1997).

[26] J. D. Eshelby, Proc. Roy. Soc. A 266, 222 (1962).

[27] K. Kang, V. V. Bulatov, and W. Cai, Proc. Nat. Acad. Sci. U.S.A. 109, 15174 (2012).

[28] J. E. Dorn and S. Rajnak, Trans. Metall. Soc. AIME 230, 1052 (1964).

[29] P. Guyot and J. E. Dorn, Can. J. Phys. 45, 983 (1967).

[30] L. Proville, L. Ventelon, and D. Rodney, Phys. Rev. B 87, 144106 (2013).

[31] M. Werner, Phys. Status Solidi A 104, 63 (1987).

[32] L. Hollang, M. Hommel, and A. Seeger, Phys. Status Solidi A 160, 329 (1997).

[33] D. Brunner, Mater. Trans. JIM. 41, 152 (2000).

[34] D. Brunner and J. Diehl, Phys. Status Solidi A 124, 155 (1991).

[35] D. Brunner and J. Diehl, Phys. Status Solidi A 124, 455 (1991).

[36] P. Giannozzi, S. Baroni, N. Bonini, M. Calandra, R. Car, C. Cavazzoni, D. Ceresoli, G. L. Chiarotti, M. Cococcioni, I. Dabo, A. Dal Corso, S. Fabris, G. Fratesi, S. de Gironcoli, R. Gebauer, U. Gerstmann, C. Gougoussis, A. Kokalj, M. Lazzeri, L. Martin-Samos, N. Marzari, F. Mauri, R. Mazzarello, S. Paolini, A. Pasquarello, L. Paulatto, C. Sbraccia, S. Scandolo, G. Sclauzero, A. P. Seitsonen, A. Smogunov, P. Umari, and R. M. Wentzcovitch, J. Phys. Condens. Matter 21, 395502 (2009).

[37] L. Ventelon, F. Willaime, C.-C. Fu, M. Heran, and I. Ginoux, J. Nucl. Mater. 425, 16 (2012).
[38] M.-C. Marinica, F. Willaime, and J.-P. Crocombette, Phys. Rev. Lett. 108, 025501 (2012).

[39] L. Dezerald, M.-C. Marinica, L. Ventelon, D. Rodney, and F. Willaime, J. Nucl. Mater. 449, 219 (2014).

[40] N. Nagasako, M. Jahnátek, R. Asahi, and J. Hafner, Phys. Rev. B 81, 094108 (2010).

[41] L. Koci, Y. Ma, A. R. Oganov, P. Souvatzis, and R. Ahuja, Phys. Rev. B 77, 214101 (2008).

[42] M. Methfessel and A. T. Paxton, Phys. Rev. B 40, 3616 (1989).

[43] D. Rodney and L. Proville, Phys. Rev. B 79, 094108 (2009).

[44] D. Rodney and J. Bonneville, in Physical Metallurgy, edited by K. Hono and D. Laughlin (Elsevier, Amsterdam, 2013).

[45] T. D. Swinburne, S. L. Dudarev, S. P. Fitzgerald, M. R. Gilbert, and A. P. Sutton, Phys. Rev. B 87, 064108 (2013).

[46] G. Henkelman, G. Jóhannesson, and H. Jónsson, Progress on Theoretical Chemistry and Physics (Kluwer Academic Publishers, Dordrecht, 2000), p. 269.

[47] R. O. Scattergood and D. J. Bacon, Philos. Mag. 31, 179 (1975).

[48] R. O. Scattergood and D. J. Bacon, Acta Metall. 30, 1665 (1982).

[49] J. P. Hirth and J. Lothe, Theory of Dislocations (Wiley, New York, 1982), p. 176.

[50] A. N. Stroh, Philos. Mag. 3, 625 (1958).

[51] J. Douin, P. Vessière, and P. Beauchamp, Philos. Mag. A 54, 375 (1986).

[52] E. Clouet, L. Ventelon, and F. Willaime, Phys. Rev. Lett. 102, 055502 (2009).

[53] J. P. Hirth and J. Lothe, Theory of Dislocations (Wiley, New York, 1982).

[54] K. A. Gschneidner, Solid State Phys. 16, 275 (1964).

[55] T. Suzuki, Y. Kamimura, and H. O. K. Kirchner, Philos. Mag. 79, 1629 (1999).

[56] S. Takeuchi, T. Hashimoto, and K. Maeda, Trans. JIM 23, 60 (1982).

[57] S. Takeuchi and K. Maeda, Acta. Metall. 25, 1485 (1977).

[58] L. Hollang, D. Brunner, and A. Seeger, Mater. Sci. Eng. A 319-321, 233 (2001)

[59] D. Brunner and V. Glebovsky, Mater. Lett. 42, 290 (2000).

[60] E. Kuramoto, Y. Aono, and K. Kitajima, Philos. Mag. 39, 717 (1979).

[61] D. Brunner and J. Diehl, Z. Metallkd. 83, 828 (1992).

[62] M. I. Mendelev, S. Han, D. J. Srolovitz, G. J. Ackland, D. Y. Sun, and M. Asta, Philos. Mag. 83, 3977 (2003).

[63] P. A. Gordon, T. Neeraj, and M. I. Mendelev, Philos. Mag. Lett. 91, 3931 (2011).

[64] B. Barvinschi, L. Proville, and D. Rodney, Modell. Simul. Mater. Sci. Eng. 22, 025006 (2014).

[65] M.-C. Marinica, L. Ventelon, M. R. Gilbert, L. Proville, S. L. Dudarev, J. Marian, G. Bencteux, and F. Willaime, J. Phys. Condens. Matter 25, 395502 (2013).

[66] A. Seeger, Mater. Sci. Eng. A 370, 50 (2004).

[67] M. R. Gilbert, P. Schuck, B. Sadigh, and J. Marian, Phys. Rev. Lett. 111, 095502 (2013).

[68] A. Satta, F. Willaime, and S. de Gironcoli, Phys. Rev. B 57, 11184 (1998).

[69] A. Satta, F. Willaime, and S. de Gironcoli, Phys. Rev. B 60, 7001 (1999). 